\title{
The cost impact to Medicare of shifting treatment of worsening heart failure from inpatient to outpatient management settings
}

This article was published in the following Dove Press journal:

ClinicoEconomics and Outcomes Research

\author{
Kathryn Fitch' \\ Jocelyn Lau' \\ Tyler Engel' \\ Joseph J Medicis ${ }^{2}$ \\ John F Mohr ${ }^{2}$ \\ William S Weintraub ${ }^{3}$ \\ 'Milliman Inc., New York, NY, USA; \\ ${ }^{2}$ scPharmaceuticals Inc., Burlington, \\ MA, USA; ${ }^{3}$ MedStar Washington \\ Hospital Center, Washington, DC, \\ USA
}

Video abstract

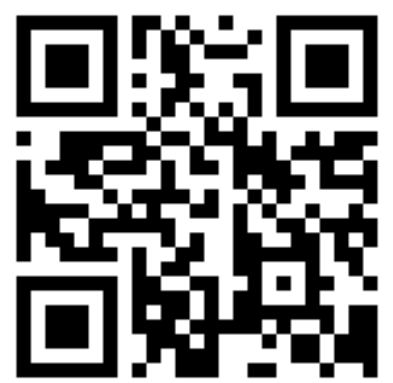

Point your SmartPhone at the code above. If you have a QR code reader the video abstract will appear. Or use: http://youtu.be/kMVCeOZcxl0

Correspondence: Kathryn Fitch Milliman Inc., I Pennsylvania Plaza, 38th floor, New York, NY I0I 19, USA

Tel +l 6464733216

Fax +I 6464733199

Email kate.fitch@milliman.com
Purpose: The aim of this study was to quantify the potential cost savings to Medicare of shifting the site of treatment for worsening heart failure (HF) from inpatient to outpatient (OP) settings for a subset of worsening HF episodes among the Medicare fee-for-service (FFS) population. Materials and methods: A cross-sectional analysis of a random 5\% sample of 2014 FFS Medicare beneficiaries was conducted. Incidence and cost of worsening HF episodes in both inpatient and OP settings were identified. These results were used to calculate cost savings associated with shifting a proportion of worsening HF episodes from the inpatient to OP settings. Results: A total of 151,908 HF beneficiaries were identified. The estimated annual cost for the treatment of worsening HF across both inpatient and OP settings ranged from US\$9.3 billion to US $\$ 17.0$ billion or $2.4 \%-4.3 \%$ of total Medicare FFS spend. The cost saving associated with shifting worsening HF treatment from inpatient hospital setting to OP settings was US\$667.5 million or $0.17 \%$ of total Medicare spend when $10 \%$ of HF admissions were targeted and $60 \%$ of targeted HF admissions were successfully shifted. The cost savings increased to US\$2.098 billion or $0.53 \%$ of total Medicare spend when $20 \%$ of HF admissions were targeted and $90 \%$ of targeted HF admissions were successfully shifted.

Conclusion: Treatment options that can shift costly hospital admissions for worsening HF treatment to less expensive OP settings potentially lead to significant cost savings to Medicare. Pursuit of OP therapy options for treating worsening HF might be considered a viable alternative. Keywords: health care resource utilization, heart failure management, hospital admission burden, administrative claims data, cost impact analysis

\section{Introduction}

Heart failure (HF), a prevalent and costly syndrome affecting $\sim 6.5$ million US adults, is projected to increase to greater than 8 million prevalent cases by $2030 .{ }^{1}$ It is projected that the percentage of the HF population that is 65 and older will increase from $62 \%$ in 2012 to $71 \%$ in $2030 .^{2}$ Total direct medical costs of HF were estimated to be US\$21 billion in 2012 and are projected to increase to US\$53 billion by 2030 with the proportion contributed by the population age $\geq 65$ years increasing from $81 \%$ to $88 \%$, making the financial burden for Medicare significant. ${ }^{2}$ The majority of the costs among the HF population ( $80 \%$ ) are associated with hospital admissions, ${ }^{2}$ and $\sim 15 \%$ of the hospital admission costs is for the treatment of worsening $\mathrm{HF}^{3}$

Worsening HF in chronic HF patients is characterized by deterioration in HF signs and symptoms, after a period of clinical stability, which requires escalation of therapy. ${ }^{4}$ For patients with worsening HF due to fluid accumulation, early intervention 
is crucial to possibly prevent hospitalization or emergency department (ED) care. Worsening HF is a key event in the natural history of HF that can be treated in the inpatient setting or outpatient (OP) settings including EDs, observation units, or physician offices. ${ }^{4}$

Currently, more than $80 \%$ of patients who present to the ED with worsening HF are admitted to the hospital. ${ }^{5}$ Many patients admitted to a hospital for the treatment of worsening HF simply need decongestion via intravenous (IV) therapy. ${ }^{6,7}$ It has been estimated that up to $50 \%$ of patients presenting to the ED with worsening HF could be stabilized and after a short period of observation could be sent home without the need for a hospital admission. ${ }^{8}$ However, the frequency at which worsening HF is treated in OP settings remains persistently low despite studies showing that ambulatory administration of IV diuretics in observation units, EDs, and clinics is a safe and effective alternative to inpatient hospital treatment for select patients. ${ }^{9-13}$ The limited availability of clinics providing IV diuretics and limited novel therapies, as well as current provider practice patterns, contributes to the low rates of treatment in OP settings.

Because inpatient visits for $\mathrm{HF}$ are more expensive than OP visits for $\mathrm{HF},{ }^{14}$ shifting treatment for worsening $\mathrm{HF}$ for selected patients from the inpatient hospital setting to OP settings could reduce costs; however, these cost savings have not been previously quantified. The objective of this study was to estimate the cost impact to the Medicare fee-for-service (FFS) population associated with shifting the treatment of a portion of worsening HF cases from the inpatient hospital setting to OP settings.

\section{Materials and methods}

\section{Data source}

We used the 2013-2014 Medicare 5\% sample (limited data set) which contains all Medicare FFS Part A and Part B paid claims (no Part D data) from a statistically balanced 5\% sample of the total Medicare population.

\section{Study population}

The denominator population for this study included beneficiaries with no health maintenance organization (HMO) enrollment and eligibility in both Part A and Part B for all months of 2013 and at least one month in 2014. HF patients were identified as individuals with one or more acute inpatient, non-acute inpatient (skilled nursing facilities and long-term rehabilitation), OP, observation, or ED claims coded with an HF ICD, ninth revision (ICD-9), diagnosis codes (428.0-428.9, 402.01, 402.11, 402.91, 404.01, 404.03,
$404.11,404.13,404.91,404.93)$ in any position on the claim in 2014. These codes, which are standard criteria used for disease identification in administrative claims data, describe HF specifically.

\section{Worsening HF episode identification}

Worsening HF hospital admissions were identified as all admissions coded with a diagnosis-related group (DRG) for HF (291, 292, or 293) or a proportion of admissions coded with HF-related DRGs (ie, cardiac and respiratory DRGs that were determined by a clinician to be clinically related to HF) 186-195, 204-208, 304-305, 308-310, and 312. DRGs are assigned based on diagnosis coding present on the claim, and the identified list of HF-related DRGs encompasses conditions that could be exacerbated by HF (eg, cardiac arrhythmia, pleural effusion, pneumonia, respiratory signs and symptoms, and others). We separately analyzed the Premier Inpatient Database to estimate the proportion of admissions for each HF-related DRG that showed evidence of treatment for worsening HF, defined by IV diuretic treatment for $>2$ days. We did not consider admissions with 1 day of treatment as worsening HF admissions, since a single dose of IV diuretic use can often be used post surgery or post transfusion, and these admissions likely would not be appropriate for treatment in OP settings. For each DRG, the proportion of HF-related DRG admissions with evidence of treatment for worsening HF in the Premier Database was applied to the corresponding DRG admissions in the Medicare 5\% sample HF population to yield the total number of HF-related admissions. These HF-related admissions, along with all the DRG 291, 292, and 293 admissions, were collectively considered hospital admissions for the treatment of worsening HF.

OP visits for the treatment of worsening HF were identified based on physician office, ED, and observation unit claims coded with a combination of HF or HF-related ICD-9 diagnosis codes $(276.69,514,518.4,518.81,518.83,518.84$, $782.3,786$ ) or J code J1940 for infused furosemide. Physician office visits were considered treatment for worsening HF if the claim was coded with 1) at least one HF or HF-related ICD-9 diagnosis code in any position of the claim and 2) J1940 for infused furosemide. ED and observation unit visits were considered treatment for worsening HF if they met any one of the following criteria: 1) coded with an HF ICD-9 diagnosis code in the primary position of the claim, 2) coded with an HF-related ICD-9 diagnosis code in the primary position of the claim and one HF ICD-9 diagnosis code in any other position of the claim, or 3) coded with an HF ICD-9 diagnosis code or HF-related ICD-9 diagnosis code in any 
position of the claim and at least one J1940 claim. The rate of episodes for the treatment of worsening HF was calculated on a per 1,000 HF population basis for each site of service.

\section{Costs}

The average cost of worsening HF admissions, which included facility and professional costs, was calculated based on a weighted average of DRGs 291, 292, and 293. The average cost of the 30 -day post-discharge period following a worsening $\mathrm{HF}$ admission was calculated as the difference between all costs incurred in the 30 days following discharge and the average 1-month cost of an HF patient. Worsening HF OP visit costs included all costs on the date of the service. The total costs associated with treatment for worsening HF included the worsening HF admission costs, 30-day post-discharge costs, and the worsening HF physician office, observation unit, and ED visit costs. These total costs were divided by the total costs of the eligible Medicare FFS denominator population to get the proportion of total Medicare FFS costs contributed by treatment for worsening HF. Costs represent allowed costs which include Medicare payment to providers plus patient cost sharing and were trended to 2017 using a 2.2\% average annual rate based on the 2017 Office of the Actuary Annual Report. ${ }^{15}$

\section{Modeling scenarios}

To estimate the total cost of treatment for worsening HF for the Medicare FFS population, we used the Medicare FFS population size and total population cost reported in the 2017 Office of the Actuary Annual Report. ${ }^{15}$ We applied the HF prevalence rate, worsening HF episode treatment rates, and average worsening HF episode treatment costs (trended to 2017) from the Medicare 5\% sample analysis to the total 2017 Medicare FFS population. To estimate potential variation around the total cost of treatment for worsening HF for the Medicare FFS population, we varied the prevalence of $\mathrm{HF}$ and the incidence and cost of worsening HF episodes identified in our Medicare 5\% sample analysis (average scenario) by $\pm 10 \%$.

To estimate the cost impact of shifting treatment for worsening HF from the inpatient hospital setting to OP settings for the Medicare FFS population, we assumed the average scenario costs as the starting cost of treatment for worsening HF. We considered three input assumptions and varied these assumptions as follows: 1) the percentage of worsening HF hospital admissions targeted for shifting; 2) the percentage of targeted admissions that successfully shift to OP settings; and 3) the distribution of shifted admissions to a physician office, ED, or observation unit. Successful shifting was defined as no admission, ED, or observation visit for the treatment of worsening HF in the 30 days after the worsening HF treatment episode.

For targeted worsening HF admissions successfully shifted to treatment in an OP setting, the cost impact assumes that the average cost of a hospital admission for worsening HF and the average 30-day post-discharge incremental cost are replaced with the average cost of treatment for the selected OP site of service. For unsuccessfully shifted target cases, the cost impact assumes that the average cost of a hospital admission for worsening HF and the average 30-day post-discharge cost are incurred. The cost savings per targeted admission reflects the total savings divided by the number of worsening HF admissions targeted for shifting to OP treatment. The framework for the cost calculation of each scenario is shown in Figure 1. Data analysis for this paper was generated using $\mathrm{SAS}^{\circledR}$ software (Version 9.4), and model calculations were performed in Microsoft Excel. This study was conducted using publicly available, patient de-identified data; therefore, an institutional review board did not conduct a review of this analysis plan. This study was conducted in accordance with the principles of the Declaration of Helsinki.

\section{Results}

\section{Baseline cost of treatment for worsening HF}

A total of 1,441,306 Medicare beneficiaries met the denominator eligibility criteria in the Medicare 5\% sample analysis, and 151,908 beneficiaries were identified with HF, yielding a prevalence of $10.5 \%$. Demographic information for this population has been previously published. ${ }^{16}$ Table 1 presents the findings of the Medicare 5\% sample (baseline scenario) including HF prevalence, annual rate of hospital admissions, and OP visits for the treatment of worsening $\mathrm{HF}$, and the average cost per worsening HF hospital admission or OP visit. Applying the Medicare 5\% sample findings to the total Medicare FFS population produces an annual Medicare FFS treatment cost for worsening HF of US $\$ 12.8$ billion or $3.3 \%$ of total annual Medicare FFS population spend. Varying the prevalence rate for $\mathrm{HF}$, the rate of hospital admissions and OP visits for the treatment of worsening $\mathrm{HF}$, and the average cost per worsening HF hospital admission and OP visit by $\pm 10 \%$, yields estimated costs for the treatment of worsening $\mathrm{HF}$ between US\$9.3 billion and US\$17.0 billion or $2.4 \%-4.3 \%$ of total Medicare FFS spend. 


\section{No shift scenario}

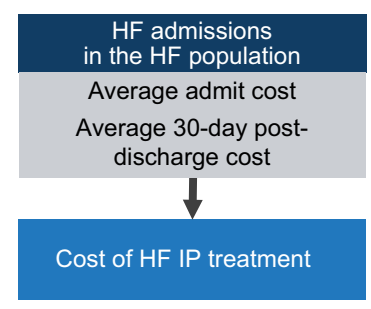

Treatment shift scenario

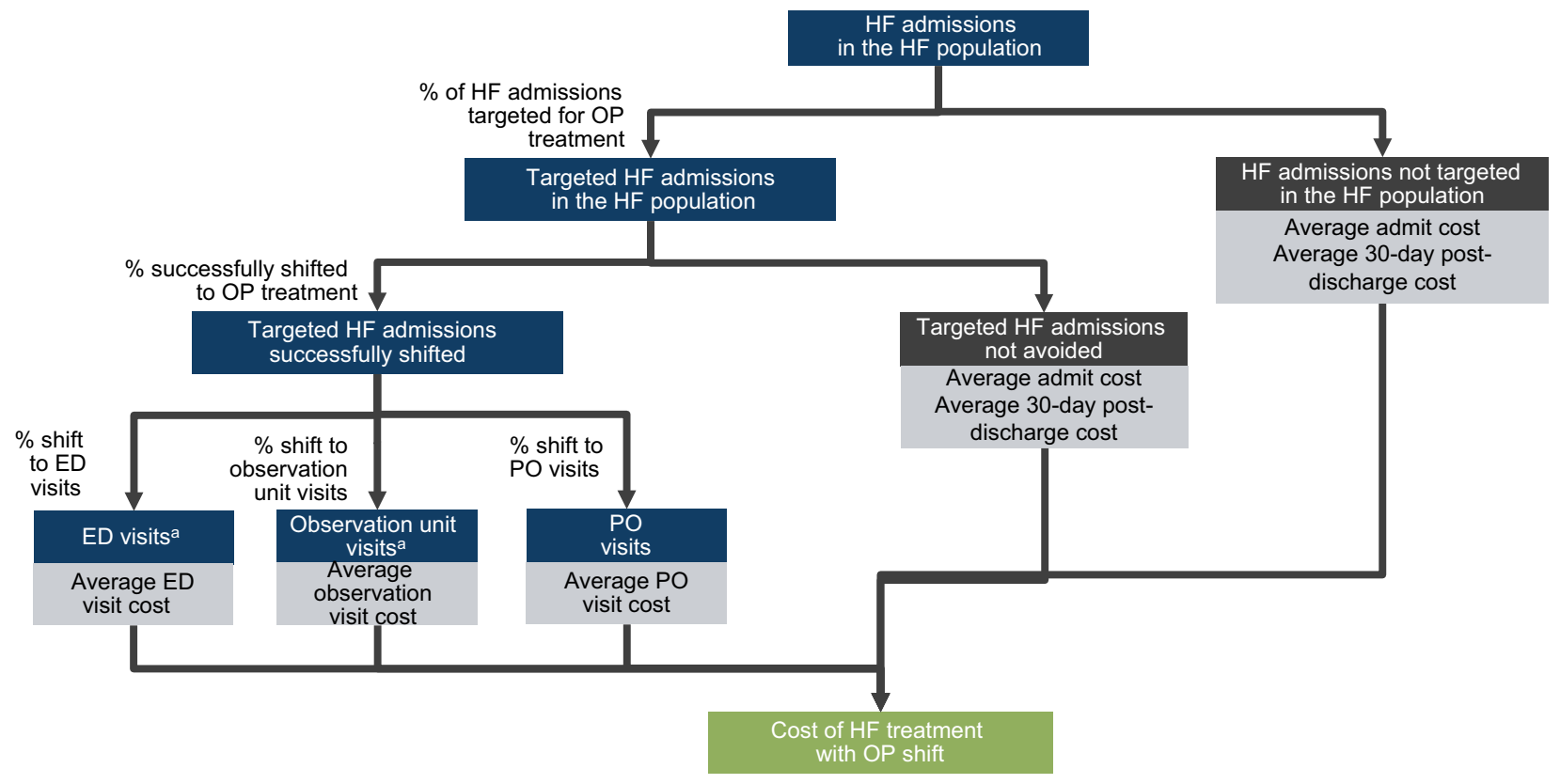

Figure I Cost impact calculation.

Note: ${ }^{\mathrm{E} E D}$ and observation unit visits are stand-alone visits that do not result in a hospital admission.

Abbreviations: ED, emergency department; HF, heart failure; IP, inpatient; OP, outpatient; PO, physician office.

Table I Medicare FFS population metrics and sensitivity testing for treatment cost of worsening HF

\begin{tabular}{|c|c|c|c|}
\hline Total Medicare FFS beneficiaries ${ }^{\mathrm{a}}$ & \multicolumn{3}{|c|}{$38,847,000$} \\
\hline Total Medicare FFS spend ${ }^{\mathrm{a}}$ & \multicolumn{3}{|c|}{$\$ 392,435,000,000$} \\
\hline Medicare FFS HF population metrics & $-10 \%$ & Baseline $^{\text {b }}$ & $+10 \%$ \\
\hline Prevalence of $\mathrm{HF}$ & $9.5 \%$ & $10.5 \%$ & $11.6 \%$ \\
\hline Rate of HF admissions per I,000 HF patients & 209.4 & 232.7 & 256.0 \\
\hline Rate of physician office visits for the treatment of worsening HF per I,000 HF patients & 8.3 & 9.3 & 10.2 \\
\hline $\begin{array}{l}\text { Rate of ED visits for the treatment of worsening HF per I,000 HF patients (not resulting in } \\
\text { admission) }\end{array}$ & 29.7 & 33.0 & 36.3 \\
\hline $\begin{array}{l}\text { Rate of observation unit visits for worsening HF treatment per I,000 HF patients (not resulting } \\
\text { in admission) }\end{array}$ & 16.1 & 17.9 & 19.7 \\
\hline Average cost per HF admission & $\$ 11,385$ & $\$ 12,650$ & $\$ 13,915$ \\
\hline Average 30-day post-discharge cost following an HF admission (net 30-day baseline HF costs) & $\$ 5,393$ & $\$ 5,992$ & $\$ 6,591$ \\
\hline Average cost per physician office visit for the treatment of worsening HF & $\$ 449$ & $\$ 499$ & $\$ 549$ \\
\hline Average cost per ED visit for the treatment of worsening HF & $\$ 1,162$ & $\$ 1,291$ & $\$ 1,420$ \\
\hline Average cost per observation unit visit for the treatment of worsening HF & $\$ 3,067$ & $\$ 3,407$ & $\$ 3,748$ \\
\hline Medicare FFS worsening HF costs & $-10 \%$ & Baseline & $+10 \%$ \\
\hline Total Medicare FFS treatment cost for hospital and OP treatment of worsening HF (in millions) & $\$ 9,299$ & $\$ 12,756$ & $\$ 16,978$ \\
\hline Percentage of total Medicare FFS population spend & $2.4 \%$ & $3.3 \%$ & $4.3 \%$ \\
\hline
\end{tabular}

Notes: All costs are presented in US\$. a 2017 Board of Trustees Report. Office of the Actuary. ${ }^{2} 2013-2014$ Medicare 5\% Sample analysis. 2014 costs have been trended to 2017 using a $2.2 \%$ annual trend factor.

Abbreviations: ED, emergency department; FFS, fee-for-service; HF, heart failure; OP, outpatient. 


\section{Worsening HF treatment cost impact associated with shifting treatment site}

While assuming all baseline scenario values as summarized in Table 1, we identified the impact on treatment costs for worsening HF associated with varying three major assumptions: the percentage of $\mathrm{HF}$ admissions targeted for shifting to treatment in OP sites $(10 \% ; 15 \%, 20 \%)$; the percentage of targeted HF admissions successfully shifted to treatment in OP sites $(60 \%, 80 \%, 90 \%)$; and the distribution of shifted admissions to physician office, $\mathrm{ED}$, or observation unit $(20 \%, 40 \%, 40 \%$; $30 \%, 45 \%, 25 \% ; 40 \%, 50 \%, 10 \%)$. Table 2 presents the total cost savings based on the variations in the three assumptions. The minimum cost savings was US\$667.5 million, or $0.17 \%$ of total Medicare spend, and the maximum cost savings was US\$2.098 billion, or $0.53 \%$ of total Medicare spend.

Figure 2 shows the savings per targeted admission based on variation in the percentage of targeted worsening HF admissions successfully shifted to OP settings and the distribution of successfully shifted hospital admissions to the three OP sites. A $10 \%$ increase in the percentage of targeted admissions successfully shifted to the OP setting is associated with a $\$ 1,666-\$ 1,746$ increase in the cost savings per targeted admission, which varies depending upon the distribution of OP settings.

Using baseline scenario values in Table 1, we performed sensitivity testing to identify the change in potential cost

Table 2 Medicare FFS cost savings associated with shifting treatment for worsening HF from inpatient hospital to OP settings

\begin{tabular}{|c|c|c|c|c|c|c|c|}
\hline \multirow{2}{*}{$\begin{array}{l}\text { Percentage } \\
\text { of admissions } \\
\text { successfully } \\
\text { shifted to op } \\
\text { settings (\%) }\end{array}$} & \multirow{2}{*}{$\begin{array}{l}\text { Distribution } \\
\text { of successfully } \\
\text { shifted admissions } \\
\text { (physician office } \\
\text { [\%]/ED [\%]/Obs } \\
{[\%] \text { ) }}\end{array}$} & \multicolumn{2}{|c|}{$\begin{array}{l}\text { I0\% of HF admissions } \\
\text { targeted }\end{array}$} & \multicolumn{2}{|c|}{$\begin{array}{l}\text { I5\% of HF admissions } \\
\text { targeted }\end{array}$} & \multicolumn{2}{|c|}{$\begin{array}{l}20 \% \text { of HF admissions } \\
\text { targeted }\end{array}$} \\
\hline & & $\begin{array}{l}\text { Total cost } \\
\text { savings } \\
\text { (millions) }\end{array}$ & $\begin{array}{l}\text { Cost } \\
\text { savings as a } \\
\text { percentage of } \\
\text { total Medicare } \\
\text { FFS spend (\%) }\end{array}$ & $\begin{array}{l}\text { Total cost } \\
\text { savings } \\
\text { (millions) }\end{array}$ & $\begin{array}{l}\text { Cost } \\
\text { savings as a } \\
\text { percentage of } \\
\text { total Medicare } \\
\text { FFS spend (\%) }\end{array}$ & $\begin{array}{l}\text { Total cost } \\
\text { savings } \\
\text { (millions) }\end{array}$ & $\begin{array}{l}\text { Cost } \\
\text { savings as a } \\
\text { percentage of } \\
\text { total Medicare } \\
\text { FFS spend (\%) }\end{array}$ \\
\hline 60 & $20 / 40 / 40$ & $\$ 667.5$ & 0.17 & $\$ 1,001.2$ & 0.26 & $\$ 1,334.9$ & 0.34 \\
\hline 60 & $30 / 45 / 25$ & $\$ 683.3$ & 0.17 & $\$ 1,025.0$ & 0.26 & $\$ 1,366.7$ & 0.35 \\
\hline 60 & $40 / 50 / 10$ & $\$ 699.2$ & 0.18 & $\$ 1,048.8$ & 0.27 & $\$ 1,398.5$ & 0.36 \\
\hline 80 & $20 / 40 / 40$ & $\$ 889.9$ & 0.23 & $\$ 1,334.9$ & 0.34 & $\$ 1,779.9$ & 0.45 \\
\hline 80 & $30 / 45 / 25$ & $\$ 911.1$ & 0.23 & $\$ 1,366.7$ & 0.35 & $\$ 1,822.2$ & 0.46 \\
\hline 80 & $40 / 50 / 10$ & $\$ 932.3$ & 0.24 & $\$ 1,398.5$ & 0.36 & $\$ 1,864.6$ & 0.48 \\
\hline 90 & $20 / 40 / 40$ & $\$ 1,001.2$ & 0.26 & $\$ 1,501.8$ & 0.38 & $\$ 2,002.4$ & 0.51 \\
\hline 90 & $30 / 45 / 25$ & $\$ 1,025.0$ & 0.26 & $\$ 1,537.5$ & 0.39 & $\$ 2,050.0$ & 0.52 \\
\hline 90 & $40 / 50 / 10$ & $\$ 1,048.8$ & 0.27 & $\$ 1,573.3$ & 0.40 & $\$ 2,097.7$ & 0.53 \\
\hline
\end{tabular}

Notes: All costs are presented in US\$. Successfully shifted assumes no readmissions or subsequent ED or Obs unit visits for worsening HF in the 30 days after the treatment date for worsening HF OP therapy. 2013-2014 Medicare 5\% sample analysis.

Abbreviations: ED, emergency department; FFS, fee-for-service; HF, heart failure; OP, outpatient; Obs, observation.

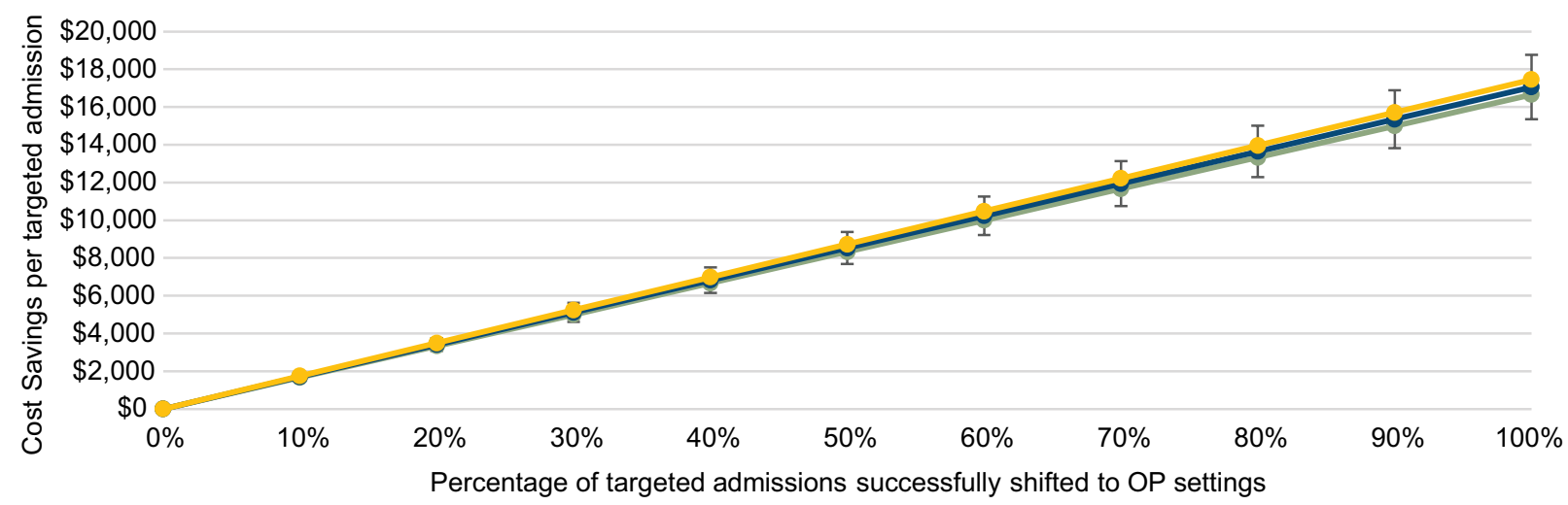

$\longrightarrow$ PO/ED/Obs: $20 \% / 40 \% / 40 \% \multimap P O / E D / O b s: 30 \% / 45 \% / 25 \% \multimap P O / E D / O b s: 40 \% / 50 \% / 10 \%$

Figure 2 Cost savings per targeted admission by the percentage of targeted admissions successfully shifted to OP settings.

Notes: All costs are presented in US\$. 2013-2014 Medicare 5\% sample analysis. Ten percent error bars are included for the distribution scenario PO/ED/Obs: 30\%/45\%/25\%. Successfully shifted assumes no readmissions or subsequent ED or Obs unit visits for worsening HF in the 30 days after the treatment date for worsening HF OP therapy. Abbreviations: ED, emergency department; HF, heart failure; Obs, observation; OP, outpatient; PO, physician office. 
impact associated with varying each input individually, as shown in Figure 3. Varying the percentage of worsening HF admissions targeted for shifting to OP treatment had the greatest impact on cost savings, reducing cost savings by $33.3 \%$ when the percentage of targeted admissions moved from $15 \%$ to $10 \%$, and increasing cost savings by $33.3 \%$ when the percentage of targeted admissions moved from $15 \%$ to $20 \%$. The variable with the next greatest impact was the percentage of targeted HF admissions successfully shifted to OP treatment which showed a $25 \%$ reduction in cost savings when the percentage of targeted admissions that were successfully shifted moved from $80 \%$ to $60 \%$ and a $12.5 \%$ increase in costs savings when the percentage of targeted admissions successfully shifted moved from $80 \%$ to $90 \%$. Varying the average cost for each type of OP visit had the smallest impact on medical cost savings.

\section{Discussion}

After a slow progression of increasing peripheral edema, worsening HF frequently generally culminates in a hospitalization for IV diuresis, even though a majority of admissions simply require decongestion, which can be performed in the OP setting. ${ }^{4}$ Barrier to OP treatment of worsening HF may include availability or access to OP HF clinics and effective medical interventions. ${ }^{5}$ The current state of worsening HF treatment remains suboptimal with over $80 \%$ of treatment occurring in an inpatient hospital setting, ${ }^{5}$ which is burdensome to the patient, the health care system, and payers. Because most of the HF population is of Medicare age, ${ }^{2}$ the cost burden of worsening HF treatment falls on Medicare in particular. This study quantified the potential cost savings for the Medicare FFS population associated with shifting treatment of worsening HF from the inpatient hospital setting to OP settings. We estimated the total cost of the treatment for worsening HF to be US\$12.7 billion and savings ranging from US\$667.5 million to US $\$ 2.097$ billion $(5.2 \%-16.4 \%$ of the cost for the treatment of worsening HF) could be realized by shifting a portion of worsening HF hospital admissions to OP settings.

Although past studies have identified the economic burden of HF among the Medicare population, they have not specifically quantified the cost of treatment for worsening HF in both inpatient hospital setting and OP settings. ${ }^{17,18}$ Other studies on HF hospitalization costs include populations that are not specific to Medicare. ${ }^{14,19,20}$ Most studies that have analyzed the cost of treatment for HF admissions have limited the admissions to DRGs 291, 292, and 293 or specific HF diagnosis codes. ${ }^{17,21}$ For instance, a separate analysis of the Medicare 5\% sample calculated the cost of HF hospitalizations, but only identified HF admissions as those with ICD-9 diagnosis codes beginning with 428 or 398.91. ${ }^{17}$ One study estimated that the number of worsening HF hospitalizations was approximately twice as high as the

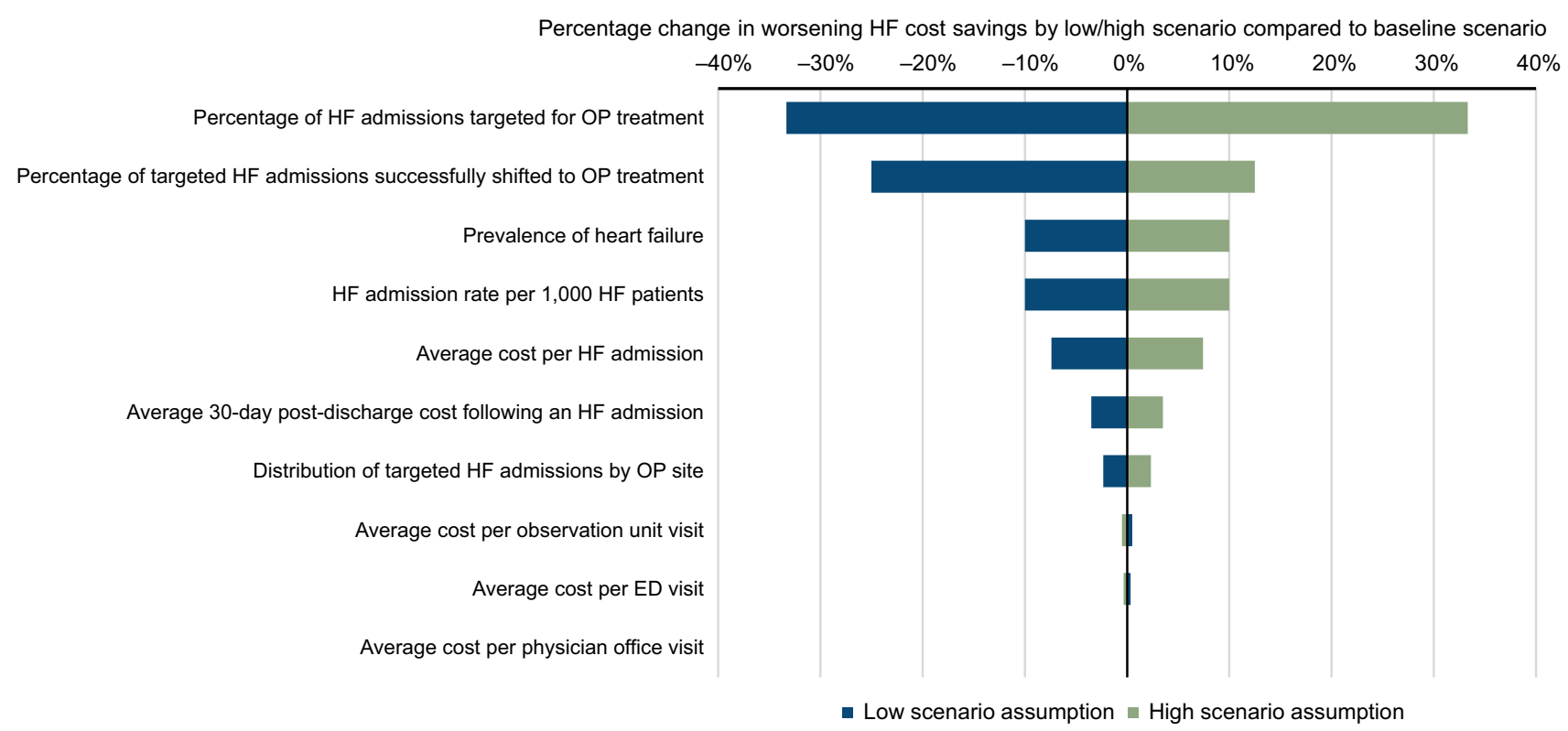

Figure 3 Sensitivity testing of the impact of selected assumptions on worsening HF cost savings.

Notes: 2013-2014 Medicare 5\% sample analysis. Low, moderate, and high percentage of worsening HF hospital admissions targeted for shifting: (10\%, I5\%, 20\%). Low, moderate, and high percentage of targeted admissions that successfully shift to OP settings $(60 \%, 80 \%, 90 \%)$. Low, moderate, and high distribution of shifted admissions to physician office, ED, or observation unit ( $20 \%, 40 \%, 40 \% ; 30 \%, 45 \%, 25 \% ; 40 \%, 50 \%, 10 \%)$. All other values varied by $\pm 10 \%$ from the baseline scenario values.

Abbreviations: ED, emergency department; HF, heart failure; OP, outpatient. 
number of hospitalizations with ICD-9 diagnosis code 428 in the primary position, ${ }^{21}$ suggesting that HF coding may not sufficiently capture all treatment for worsening HF. Because many respiratory and cardiac DRG admissions beyond the typical HF DRGs 291, 292, and 293 admissions may include diuretic treatment for worsening HF, we used the Premier Inpatient Database to identify the proportion of several respiratory and cardiac DRGs with diuretic use and applied these rates to the Medicare 5\% sample to identify additional admissions for treatment for worsening HF that may be missed in other analyses. This methodology recognizes that only a fraction of the selected respiratory and cardiac DRGs represent worsening $\mathrm{HF}$ admissions that could be shifted to other sites of care. Furthermore, our criteria for the identification of worsening HF in OP settings also incorporated diuretic use in combination with HF diagnosis coding. In using these criteria for the identification of worsening HF episodes, we found that worsening HF treatment costs represent 3.3\% of the total Medicare FFS spend.

As expected, this study identified significantly higher average costs for the treatment of worsening HF in the inpatient hospital setting vs OP settings which is consistent with other analyses of HF costs by site of service. ${ }^{14}$ These cost differences present opportunity for savings when more expensive hospital admissions for worsening HF are appropriately shifted to treatment in OP settings. We estimated the range of potential cost savings by varying assumptions regarding the rate of worsening HF admissions targeted for OP treatment and the rate of targeted worsening HF admissions successfully shifted to OP sites of treatment. Prior research has estimated that $\sim 10 \%-20 \%$ of ED presentations for HF are discharged directly home, ${ }^{8}$ suggesting that physicians determined these to be low-risk visits that did not require more intensive treatment in the inpatient hospital setting. Thus, we tested three values within this range, $10 \%, 15 \%$, $20 \%$, as an approximation for the percentage of visits that could be targeted for treatment in OP settings. The range of values for the percentage of targeted admissions that are successfully treated $(60 \%, 80 \%, 90 \%)$ assumes that the majority of admissions that are targeted for shifting to OP settings would successfully shift. The percentage of targeted HF admissions and the success of shifting an admission to the OP setting would be dependent on a particular community's ambulatory care capabilities; therefore, the estimated impact of cost shifting would need to be individualized to that community. The distribution of successfully shifted admissions to physician office, ED, or observation units reflects various scenarios in which admissions are shifted to sites that require more direct clinical management (a higher proportion of ED and observation unit visits) or less clinical management (a higher proportion of physician office visits).

Congestion is a marker of adverse prognosis; thus, a number of remote monitoring devices have been developed to track HF patients when they are not under active medical treatment. These devices use various technologies, including bioimpedance, wireless pulmonary artery pressure monitors, and dielectric sensing. These devices have shown to reduce the incidence of HF mortality and inpatient admissions and represent a useful tool in tracking HF patients. ${ }^{22,23}$ In this context, to be effective, monitoring must also be complemented with effective OP management strategies in the event that disease progression cannot be avoided. Evidence suggests that the OP setting can be an appropriate and effective place for worsening HF treatment resulting in better patient outcomes. ${ }^{24}$ An analysis of IV diuretic HF treatment among 60 patients in an OP HF clinic found notable changes in urine output and weight loss, suggesting this may be a valid alternative to hospitalization for selected HF patients. ${ }^{9}$ Other studies conducted in OP HF clinics reached similar conclusions regarding the safety of OP treatment for worsening HF. ${ }^{10-13}$ OP treatment may also be associated with a reduction in subsequent hospital admissions. ${ }^{25,26}$ At a same-day access HF clinic launched by Duke University Hospital, a 10\% reduction in 30-day readmissions was observed out of over 3,000 visits over the course of 3 years. ${ }^{26}$ On the other hand, a retrospective multicenter study found readmission rates higher for HF treatment in observation units compared to inpatient hospital treatment, suggesting the need for improvements in the process of determining which worsening $\mathrm{HF}$ patients are most appropriate for OP treatment. ${ }^{27}$

Despite evidence of the safety and effectiveness of OP worsening HF treatment, the majority of treatment for worsening HF occurs in the inpatient hospital setting. This happens despite the fact that when decongestion via IV diuretics is the only necessary intervention, symptom relief can be achieved relatively quickly with most patients not requiring additional treatment. ${ }^{8}$ Indeed, the same-day access clinic at Duke University Hospital was initiated given that over half of HF admissions admitted from the ED were low risk requiring only decongestion. ${ }^{26}$ Same-day access clinics for OP diuresis have been shown to be a safe and potentially cost-effective strategy to reduce admissions, yet few institutions have adopted such clinics due to the complexities of implementation. Inpatient admission may be necessary for severe cases of worsening HF or when comorbidities occur, yet hospitalization for decongestion alone occurs frequently 
due to the lack of accessible OP treatment alternatives. ${ }^{4} \mathrm{OP}$ treatment of worsening HF has the potential to reduce the economic and public health burden associated with inpatient hospital treatment, representing a major opportunity for therapeutic advancements. ${ }^{4}$ One option is the subcutaneous administration of a novel $\mathrm{pH}$ neutral formulation of furosemide via a wearable subcutaneous delivery system, which has been shown to provide equivalent diuresis and natriuresis compared to IV-administered furosemide for worsening $\mathrm{HF}^{28-30}$ and may provide an opportunity for the management of worsening HF treatment in the OP setting.

This study is subject to several limitations. Claims coding may not fully capture all patients with HF or all episodes for the treatment of worsening HF. Without clinical data to validate our identification of episodes to treat worsening HF, we may overor underestimate the total number of worsening HF episodes. To improve our estimate of the total number of worsening HF admissions, we incorporated a metric of the proportion of cardiac and respiratory DRG admissions that had diuretic use from the Premier Inpatient Database. However, differences in the population characteristics of the Premier Inpatient Database and the Medicare 5\% sample may have led to an overestimate of the proportion of worsening HF admissions, particularly if the Premier Inpatient Database has fewer admissions requiring longer diuretic use compared to the Medicare 5\% sample population.

In estimating the cost savings associated with shifting treatment of worsening HF from the inpatient hospital setting to OP settings, our assumptions for the proportion of worsening HF admissions to target and the proportion of those targeted that would be successfully shifted to OP settings were based on several studies mentioned previously. In clinical practice, these rates may vary based on changes in physician practice patterns or improvements in overall HF management that can reduce the total number of HF admissions that can be targeted for shifting to OP settings. In addition, our cost savings assume that hospital admissions for worsening HF that are targeted and shifted to OP settings reflect the average worsening HF admission cost and 30-day post-discharge costs, whereas the average cost of the worsening HF admissions that are shifted may be of a lower severity and possibly lower cost, which would overestimate the cost savings. Furthermore, the cost savings calculated do not incorporate any additive cost associated with OP therapy that would likely be introduced to enhance the shift to OP treatment of worsening HF. Costs of new technologies or interventions implemented for each targeted admission would need to be considered in the context of the cost savings per targeted admission as shown in Figure 2. Finally, this study was conducted using the Medicare
5\% sample FFS population and the results may not be generalizable to other HF populations such as Medicare advantage or commercial populations.

\section{Conclusion}

Currently, there is a subset of patients being hospitalized for worsening HF that could potentially be treated in lower cost OP settings. With the availability of efficacious OP therapy options for treating worsening HF, OP therapy can become a viable alternative to be considered throughout the course of a patient's worsening HF progression. As the baby boomers continue to age into Medicare, the cost burden to Medicare associated with treatment for worsening HF will grow, highlighting the need for more cost-efficient alternatives for the treatment of worsening HF. Treatment options that can shift costly hospital admissions for the treatment of worsening HF to less expensive OP settings can potentially lead to significant cost savings to Medicare.

\section{Disclosure}

Kathryn Fitch is a Principal of the New York Health Practice of Milliman, Inc. Jocelyn Lau and Tyler Engel are employees of Milliman, Inc. Kathryn Fitch received consulting fees from scPharmaceuticals, Inc. to conduct this study. Dr. William S Weintraub is the Director of Outcomes Research at MedStar Washington Hospital Center. Drs. Joseph J Medicis and John F Mohr are employees and stock holders of scPharmaceuticals. Dr. Joseph J Medicis is Senior Director, Medical Affairs, at scPharmaceuticals, Inc. and Dr. John F Mohr is Vice President, Clinical Development and Medical Affairs at scPharmaceuticals, Inc. The authors report no other conflicts of interest in this work.

\section{References}

1. Benjamin EJ, Blaha MJ, Chiuve SE, et al. Heart disease and stroke statistics-2017 update: a report from the American Heart Association. Circulation. 2017;135(10):e146-e603.

2. Heidenreich PA, Albert NM, Allen LA. Forecasting the impact of heart failure in the United States: a policy statement from the American Heart Association. Circ Hear Fail. 2014;6(3):606-619.

3. Whellan DJ, Greiner MA, Schulman KA, Curtis LH. Costs of inpatient care among Medicare beneficiaries with heart failure, 2001 to 2004. Circ Cardiovasc Qual Outcomes. 2010;3(1):33-40.

4. Greene SJ, Mentz RJ, Felker GM. Outpatient worsening heart failure as a target for therapy: a review. JAMA Cardiol. 2018;3(3):252-258.

5. Storrow AB, Jenkins CA, Self WH, et al. The burden of acute heart failure on U.S. emergency departments. JACC Heart Fail. 2014;2(3):269-277.

6. Adams KF, Fonarow GC, Emerman CL, et al. Characteristics and outcomes of patients hospitalized for heart failure in the United States: rationale, design, and preliminary observations from the first 100,000 cases in the Acute Decompensated Heart Failure National Registry (ADHERE). Am Heart J. 2005;149(2):209-216. 
7. Ambrosy AP, Fonarow GC, Butler J, et al. The global health and economic burden of hospitalizations for heart failure: lessons learned from hospitalized heart failure registries. J Am Coll Cardiol. 2014;63(12):1123-1133.

8. Collins SP, Pang PS, Fonarow GC, Yancy CW, Bonow RO, Gheorghiade M. Is hospital admission for heart failure really necessary?: the role of the emergency department and observation unit in preventing hospitalization and rehospitalization. J Am Coll Cardiol. 2013;61(2):121-126.

9. Buckley LF, Carter DM, Matta L, et al. Intravenous diuretic therapy for the management of heart failure and volume overload in a multidisciplinary outpatient unit. JACC Heart Fail. 2016;4(1):1-8.

10. Ryder M, Murphy NF, Mccaffrey D, O’Loughlin C, Ledwidge M, Mcdonald K. Outpatient intravenous diuretic therapy; potential for marked reduction in hospitalisations for acute decompensated heart failure. Eur J Heart Fail. 2008;10(3):267-272.

11. Banerjee P, Tanner G, Williams L. Intravenous diuretic day-care treatment for patients with heart failure. Clin Med. 2012;12(2):133-136.

12. Hebert K, Dias A, Franco E, Tamariz L, Steen D, Arcement LM. Open access to an outpatient intravenous diuresis program in a systolic heart failure disease management program. Congest Heart Fail. 2011;17(6):309-313.

13. Lazkani M, Ota KS. The role of outpatient intravenous diuretic therapy in a transitional care program for patients with heart failure: a case series. J Clin Med Res. 2012;4(6):434-438.

14. Voigt J, Sasha John M, Taylor A, Krucoff M, Reynolds MR, Michael Gibson C. A reevaluation of the costs of heart failure and its implications for allocation of health resources in the United States. Clin Cardiol. 2014;37(5):312-321.

15. The Board of Trustees Federal Hospital Insurance and Federal Supplementary Medical Insurance Trust Funds. 2017 Annual Report of the Boards of Trustees of the Federal Hospital Insurance and Federal Supplementary Medical Insurance Trust Funds; 2017.

16. Fitch K, Engel T, Lau J. The Cost Burden of Worsening Heart Failure in the Medicare Fee For Service Population: An Actuarial Analysis. Seattle: Milliman; 2017.

17. Kilgore M, Patel HK, Kielhorn A, Maya JF, Sharma P. Economic burden of hospitalizations of Medicare beneficiaries with heart failure. Risk Manag Healthc Policy. 2017;10:63-70.

18. Fitch K, Pelizzari PM, Pyenson B. Inpatient utilization and costs for Medicare fee-for-service beneficiaries with heart failure. Am Health Drug Benefits. 2016;9(2):96-104.
19. Wang G, Zhang Z, Ayala C, Wall HK, Fang J. Costs of heart failurerelated hospitalizations in patients aged 18 to 64 years. Am J Manag Care. 2010;16(10):769-776.

20. Titler MG, Jensen GA, Dochterman JM, et al. Cost of hospital care for older adults with heart failure: medical, pharmaceutical, and nursing costs. Health Serv Res. 2008;43(2):635-655.

21. Agarwal SK, Wruck L, Quibrera M, et al. Temporal trends in hospitalization for acute decompensated heart failure in the United States, 1998-2011. Am J Epidemiol. 2016;183(5):462-470.

22. Klersy C, de Silvestri A, Gabutti G, Regoli F, Auricchio A. A metaanalysis of remote monitoring of heart failure patients. $\mathrm{J} \mathrm{Am}$ Coll Cardiol. 2009;54(18):1683-1694.

23. Martirosyan M, Caliskan K, Theuns D, Szili-Torok T. Remote monitoring of heart failure: benefits for therapeutic decision making. Expert Rev Cardiovasc Ther. 2017;15(7):503-515.

24. Fonarow GC, Albert NM, Curtis AB, et al. Improving evidence-based care for heart failure in outpatient cardiology practices: primary results of the Registry to Improve the Use of Evidence-Based Heart Failure Therapies in the Outpatient Setting (IMPROVE HF). Circulation. 2010;122(6):585-596.

25. Makadia S, Simmons T, Augustine S, et al. The diuresis clinic: a new paradigm for the treatment of mild decompensated heart failure. Am J Med. 2015;128(5):527-531.

26. Devore AD, Allen LA, Eapen ZJ. Thinking outside the box: treating acute heart failure outside the hospital to improve care and reduce admissions. J Card Fail. 2015;21(8):667-673.

27. Masri A, Althouse AD, Mckibben J, et al. Outcomes of heart failure admissions under observation versus short inpatient stay. J Am Heart Assoc. 2018;7(3):e007944.

28. Gilotra NA, Princewill O, Marino B, et al. Efficacy of intravenous furosemide versus a novel, $\mathrm{pH}$-neutral furosemide formulation administered subcutaneously in outpatients with worsening heart failure. JACC Heart Fail. 2018;6(1):65-70.

29. Sica DA, Muntendam P, Myers RL, et al. Subcutaneous furosemide in heart failure: pharmacokinetic characteristics of a newly buffered solution. JACC Basic Transl Sci. 2018;3(1):25-34.

30. Myers RL, Udelson J, Cornelius B, et al. On-body performance of the sc2Wear ${ }^{\mathrm{TM}}$ infusor for subcutaneous (SC) administration of a novel formulation of furosemide in patients with heart failure (HF). J Card Fail. 2017;23(8):S66.
ClinicoEconomics and Outcomes Research

\section{Publish your work in this journal}

ClinicoEconomics and Outcomes Research is an international, peerreviewed open-access journal focusing on health technology assessment, pharmacoeconomics and outcomes research in the areas of diagnosis, medical devices, and clinical, surgical and pharmacological intervention. The economic impact of health policy and health systems
Submit your manuscript here: https://www.dovepress.com/clinicoeconomics-and-outcomes-research-jour

organization also constitute important areas of coverage. The manuscript management system is completely online and includes a very quick and fair peer-review system, which is all easy to use. Visit http://www.dovepress.com/testimonials.php to read real quotes from published authors. 